\title{
Mastering Windows: Improving Reconstruction
}

\author{
Thomas Theußl \\ Helwig Hauser \\ Eduard Gröller *
}

Institute of Computer Graphics

Vienna University of Technology
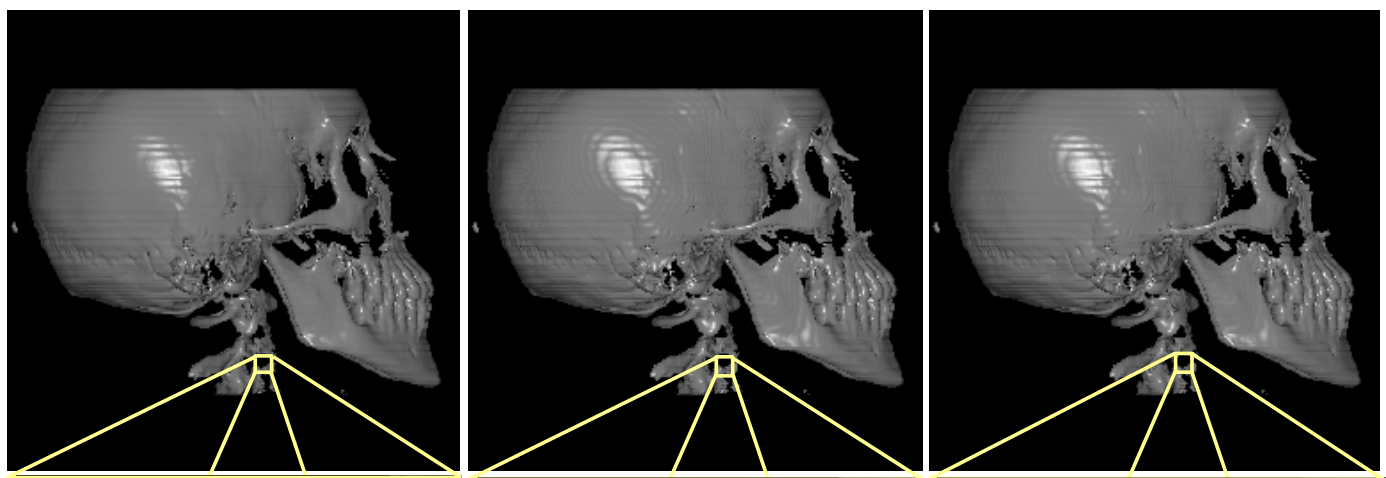

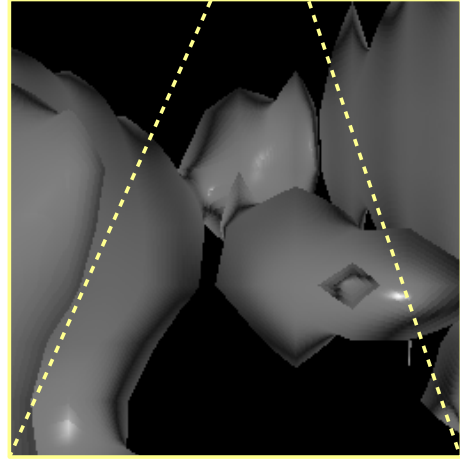

(a)

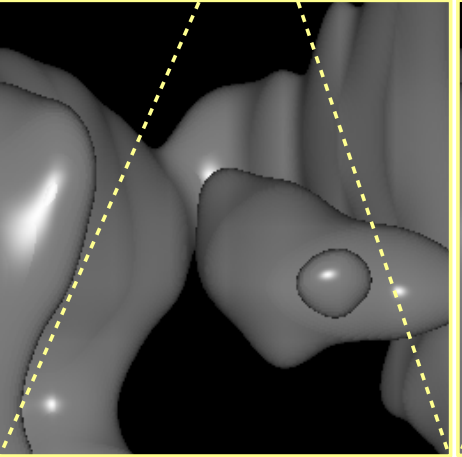

(b)

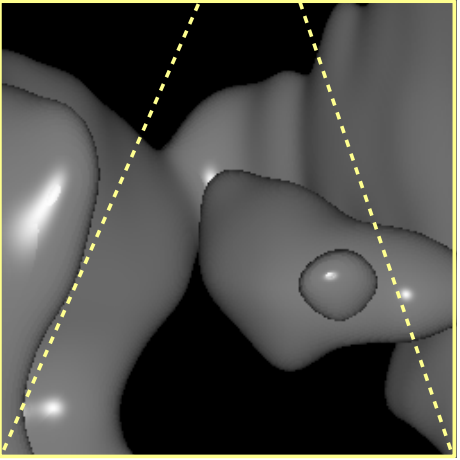

(c)

Figure 1: A CT scan of a head reconstructed with (a) linear interpolation and central differences with linear interpolation, (b) Catmull-Rom spline and derivative and (c) Kaiser windowed sinc and cosc of width three with numerically optimal parameters.

\section{Abstract}

Ideal reconstruction filters, for function or arbitrary derivative reconstruction, have to be bounded in order to be practicable since they are infinite in their spatial extent. This can be accomplished by multiplying them with windowing functions. In this paper we discuss and assess the quality of commonly used windows and show that most of them are unsatisfactory in terms of numerical accuracy. The best performing windows are Blackman, Kaiser and Gaussian win-

\footnotetext{
*\{theussl,helwig,meister $@$ @cg.tuwien.ac.at
}

dows. The latter two are particularly useful since both have a parameter to control their shape, which, on the other hand, requires to find appropriate values for these parameters. We show how to derive optimal parameter values for Kaiser and Gaussian windows using a Taylor series expansion of the convolution sum. Optimal values for function and first derivative reconstruction for window widths of two, three, four and five are presented explicitly.

Keywords: ideal reconstruction, windowing, frequency response, Taylor series expansion

\section{Introduction}

Reconstruction is a fundamental process in volume visualization. Modalities like CT or MRI scanners provide discrete data sets of continuous real objects, for instance patients in medical visualization. The function in between sample points has to be reconstructed.

It is well known that a band-limited and properly sampled function can be perfectly reconstructed by convolving the samples with the ideal function reconstruction filter. Similarly, derivatives of the function can, due to linearity of con- 
volution and derivation, directly be reconstructed by convolving the samples with the corresponding derivative of the ideal reconstruction filter. The first derivative of a threedimensional function, the gradient, can be interpreted as a normal to an iso-surface passing through the point of interest. This gradient is usually used for shading or classification and therefore the quality of gradient reconstruction strongly affects the visual appearance of the final image.

From signal processing theory we know that the ideal function reconstruction filter is the sinc filter.

$$
\operatorname{sinc}(x)=\left\{\begin{array}{cl}
\frac{\sin \pi x}{\pi x} & \text { if } x \neq 0 \\
1 & \text { if } x=0
\end{array}\right.
$$

The first derivative of the sinc filter, called cosc filter,

$$
\operatorname{cosc}(x)=\left\{\begin{array}{cl}
\frac{\cos (\pi x)-\operatorname{sinc}(x)}{x} & \text { if } x \neq 0 \\
0 & \text { if } x=0
\end{array}\right.
$$

consequently is the ideal first derivative reconstruction filter [1]. In general, the ideal reconstruction filter of the $n^{\text {th }}$ derivative of a function is the $n^{\text {th }}$ derivative of the sinc function.

However, since these filters are infinite in their spatial extent they are impracticable. Simple truncation causes severe ringing artifacts, as can be seen in Fig. 6, which shows the Marschner-Lobb data set [6] reconstructed with linear interpolation and central differences and linear interpolation (Fig. 6a), the Catmull-Rom spline and derivative (Fig. 6b) and the truncated cosc of width three (Fig. 6c). (for high resolution images please refer to the web page of this project [15]). We will investigate and explain the cause of this bad result in frequency domain in Chapter 3 and in spatial domain in Chapter 4.

To reduce artifacts originating from truncation the ideal reconstruction filters can be multiplied with appropriate functions which drop off more smoothly at the edges. We will discuss some of the more commonly used of these functions, which are adversely called windows. We show that most of them are unsuitable for reconstruction, especially for higher order derivative reconstruction, although some, for example Hamming [5] and Lanczos [16] windows, are reported to be commonly used.

\section{Previous work}

Turkowski [16] used windowed sinc functions for image resampling. He found the Lanczos window superior in terms of reduction of aliasing, sharpness, and minimal ringing as conclusion of an empirical experiment.

Marschner and Lobb [6] used a cosine-bell windowed sinc (often called Hann window) and found it superior to the entire family of cubic reconstruction filters. According to their metric there is always a windowed sinc with better smoothing and post-aliasing properties. Machiraju and Yagel [5] use a Hamming windowed sinc without further explanation of their choice. However, we found the Hamming window of not being optimal. Recently, Meijering et al. [7] performed a purely quantitative comparison of eleven windowed sinc functions. They found the Welch, Cosine, Lanczos and Kaiser windows yielding the best results and noted that the truncated sinc was one of the worst performing reconstruction filters.

Goss [2] extended the idea of windowing the ideal reconstruction filter to derivative reconstruction. The parameter of the Kaiser window controls the smoothing characteristics of the reconstructed function. Goss uses the windowed cosc only on sampling points, in between some interpolation has to be performed which is not stated explicitly.

Most researchers assess the quality of reconstruction filters in frequency domain, whereas Möller et. al [10] propose a purely numerical method operating in spatial domain. We will review this method in Chapter 4 as it will allow us to derive optimal parameters, in terms of numerical accuracy, for the Kaiser and Gaussian windows.

\section{Windowing ideal reconstruction filters}

The windows investigated in this work are

- The rectangular window or box function

$$
\Pi_{\tau}(x)= \begin{cases}1 & \text { if }|x| \leq \tau \\ 0 & \text { else }\end{cases}
$$

which performs pure truncation.

- The Bartlett window, which is actually just a tent function

$$
\operatorname{Bartlett}_{\tau}(x)=\left\{\begin{array}{cl}
1-\frac{|x|}{\tau} & \text { if }|x|<\tau \\
0 & \text { else }
\end{array}\right.
$$

- The Welch window:

$$
\operatorname{Welch}_{\tau}(x)=\left\{\begin{array}{cl}
1-\left(\frac{x}{\tau}\right)^{2} & |x|<\tau \\
0 & \text { else }
\end{array}\right.
$$

- The Parzen window

$$
\operatorname{Parzen}(x)=\frac{1}{4}\left\{\begin{array}{cl}
4-6|x|^{2}+3|x|^{3} & 0 \leq|x|<1 \\
(2-|x|)^{3} & 1 \leq|x|<2 \\
0 & \text { else }
\end{array}\right.
$$

is a piece-wise cubic approximation of the Gaussian window with extend two. Although its width is not directly adjustable it can, of course, be scaled to every desired extend.

- The Hann window (due to Julius van Hann, often wrongly referred to as Hanning window [17], sometimes just cosine bell window) and Hamming window, which are quite similar, they only differ in the choice of one parameter $\alpha$ :

$$
\mathrm{H}_{\tau, \alpha}(x)=\left\{\begin{array}{cl}
\alpha+(1-\alpha) \cos \left(\pi \frac{x}{\tau}\right) & |x|<\tau \\
0 & \text { else }
\end{array}\right.
$$

with $\alpha=\frac{1}{2}$ being the Hann window and $\alpha=0.54$ the Hamming Window.

- The Blackman window, which has one additional cosine term as compared to the Hann and Hamming window.

$$
\operatorname{Blackman}_{\tau}(x)=\left\{\begin{array}{cl}
0.42+\frac{1}{2} \cos \left(\pi \frac{x}{\tau}\right)+ & \\
0.08 \cos \left(2 \pi \frac{x}{\tau}\right) & |x|<\tau \\
0 & \text { else }
\end{array}\right.
$$

- The Lanczos window, which is the central lobe of a sinc function scaled to a certain extend.

$$
\operatorname{Lanczos}_{\tau}(x)=\left\{\begin{array}{cl}
\frac{\sin \left(\pi \frac{x}{\tau}\right)}{\pi \frac{x}{\tau}} & |x|<\tau \\
0 & \text { else }
\end{array}\right.
$$



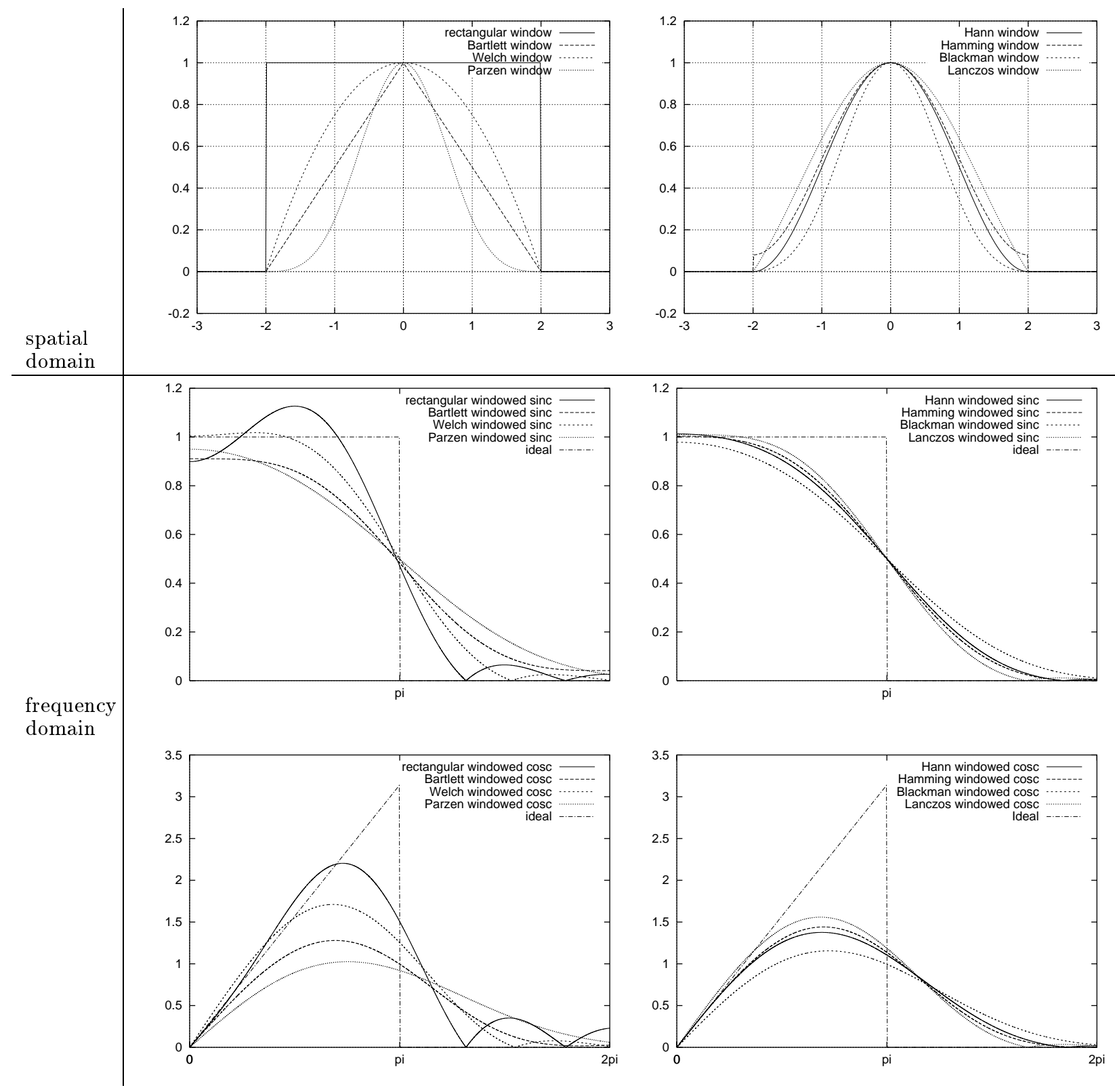

Figure 2: Rectangular, Bartlett, Welch, Parzen, Hann, Hamming, Blackman and Lanczos windows of width two on top, below the frequency responses of correspondingly windowed sinc and cosc functions.

- The Kaiser window [3], which has an adjustable parameter $\alpha$ which controls how steeply it approaches zero at the edges. It is defined by

$$
\operatorname{Kaiser}_{\tau, \alpha}(x)=\left\{\begin{array}{cl}
\frac{I_{0}\left(\alpha \sqrt{1-(x / \tau)^{2}}\right)}{I_{0}(\alpha)} & |x| \leq \tau \\
0 & \text { else }
\end{array}\right.
$$

where $I_{0}(x)$ is the zeroth order modified Bessel function [13]. The higher $\alpha$ gets the narrower becomes the Kaiser window.

- and we can also use a truncated Gaussian function as window. It is in its general form defined by

$$
\operatorname{Gauss}_{\tau, \sigma}(x)=\left\{\begin{array}{cl}
2^{-\left(\frac{x}{\sigma}\right)^{2}} & |x|<\tau \\
0 & \text { else }
\end{array}\right.
$$

with $\sigma$ being the standard deviation. The higher $\sigma$ gets, the wider the Gaussian window becomes and, on the other hand, the more severe gets the truncation.

All these windows, except Kaiser and Gaussian windows, are depicted in Fig. 2 on top, the frequency responses of correspondingly windowed sinc (with window width two) in the middle row and windowed cosc in the bottom row. Since function reconstruction filters are even functions and first derivative filters are odd functions, the power spectra, as 

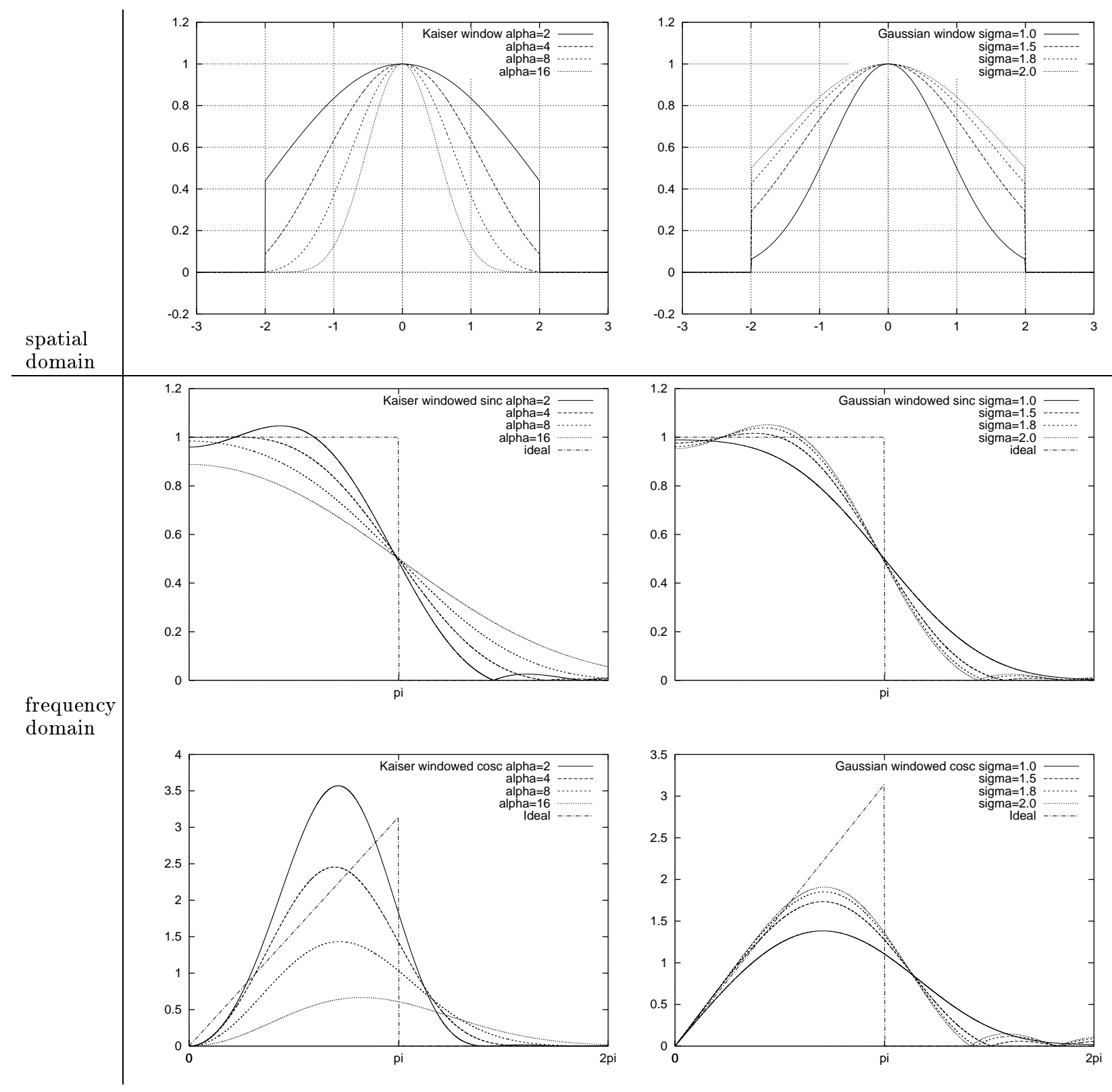

Figure 3: Kaiser and Gaussian windows of width two with varying parameters on top, below again the frequency responses of correspondingly windowed sinc and cosc functions.

depicted in Figs. 2 and 3, actually are the absolute values of the real respectively the imaginary part of the Fourier transform of the filters. Some frequency responses, e.g., the rectangular or Lanczos windowed sinc and cosc, actually assume negative values, which means that the phases of certain frequencies are reverted. This, of course, causes severe artifacts. Consequently, these windows are not suitable for reconstruction purposes.

Kaiser and Gaussian windows, with varying parameters, are depicted in Fig. 3 on top, the frequency responses of correspondingly windowed sinc (with window width two) in the middle row and windowed cosc in the bottom row. These plots show that the parameters $\alpha$ and $\sigma$ of the Kaiser and Gaussian windows directly affect the shape of their frequency responses. The frequency responses for some values of $\alpha$ and $\sigma$ again become negative, which implies that these values should be chosen carefully.

\section{Numerical analysis}

Möller et al. [10] introduced an elegant method to assess the quality of a reconstruction filter, based solely on numerical accuracy. We will shortly review this method as it is crucial for our purpose. It will allow us to quantitatively assess filter quality in spatial domain and to derive numerically optimal parameters for Kaiser and Gaussian windows.

Reconstructing a function $f(x)$ or derivatives thereof from its discrete samples $f[k]$ is done by convolving it with a con- 

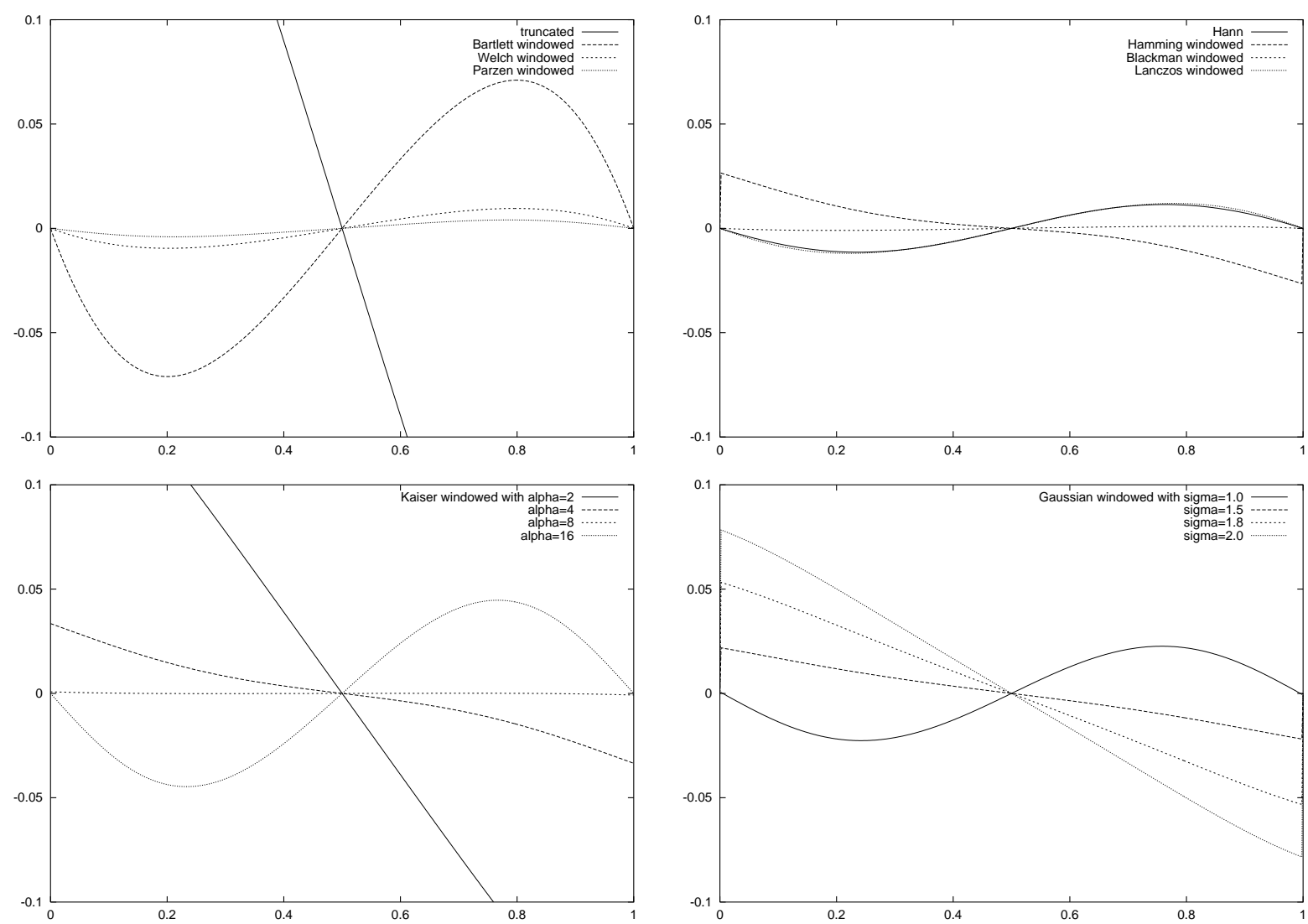

Figure 4: Coefficient plot of Taylor series expansion for windowed cosc filters with width three.

tinuous filter:

$$
f_{r}(x)=\sum_{k=-\infty}^{k=\infty} f[k] \cdot h(x-k)
$$

$f_{r}$ denotes the reconstructed function (which can be the function itself or an arbitrary derivative, depending on the reconstruction filter $h$ ). Such a convolution is a weighted average of the samples which is finite when a finite reconstruction filter is used.

$f[k]$ can now be expanded as a Taylor series in $f$ about $x$ assuming that the first $N+1$ derivatives of $f(x)$ exist:

$$
f[k]=\sum_{n=0}^{N} \frac{f^{(n)}(x)}{n !}(k-x)^{n}+\frac{f^{(N+1)}\left(\xi_{k}\right)}{(N+1) !}(k-x)^{(N+1)}
$$

where $\xi_{k} \in[x, k]$. Substituting this in Eq. 12 and reordering the terms in order of the derivatives yields

$$
f_{r}(x)=\sum_{n=0}^{N} a_{n}(x) f^{(n)}(x)+r_{N}(x)
$$

with the coefficients

$$
a_{n}(x)=\frac{1}{n !} \sum_{k=-\infty}^{k=\infty}(k-x)^{n} \cdot h(x-k)
$$

and the remainder term

$$
r_{N}(x)=\frac{1}{(N+1) !} \sum_{k=-\infty}^{k=\infty} f^{(N+1)}\left(\xi_{k}\right)(k-x)^{(N+1)} h(x-k)
$$

Eq. 14 shows that each derivative of the function has an associated coefficient. If a particular derivative of the function $f(x)$ has to be reconstructed, the corresponding coefficient has to be one and all others have to be zero, in the ideal case. For practicable filters, only the first few coefficients will obey this scheme. The more coefficients agree with this scheme, the more numerically accurate the filter is. If the $n^{\text {th }}$ coefficient of a $n^{\text {th }}$ derivative filter is different from one, the filter has to be normalized by dividing by $a_{n}$ [9]. In the following discussion this normalization step is assumed to be performed when necessary and not stated explicitly.

The coefficients $a_{n}$ only depend on the filter. This allows a classification where all filters which $N^{t h}$ coefficient, beyond the coefficient corresponding to the kind of derivative to be reconstructed (which has to be one) is different from zero, belong to one class [9]. Filters in class $N-1$ are called $N^{\text {th }}$ degree error filters $(N-E F)$. They can exactly reconstruct a polynomial of degree $N-1$ or lower. Further assessment of filters in one class could be accomplished with the remainder term $r_{N}$. Note, that the coefficients $a_{n}$ actually are functions defined between zero and one, i.e., depending on the distance to the next sampling point [9].

We use this concept to analyze the effects of windowing the ideal reconstruction filters. In Fig. 4 the coefficient $a_{0}$ is 

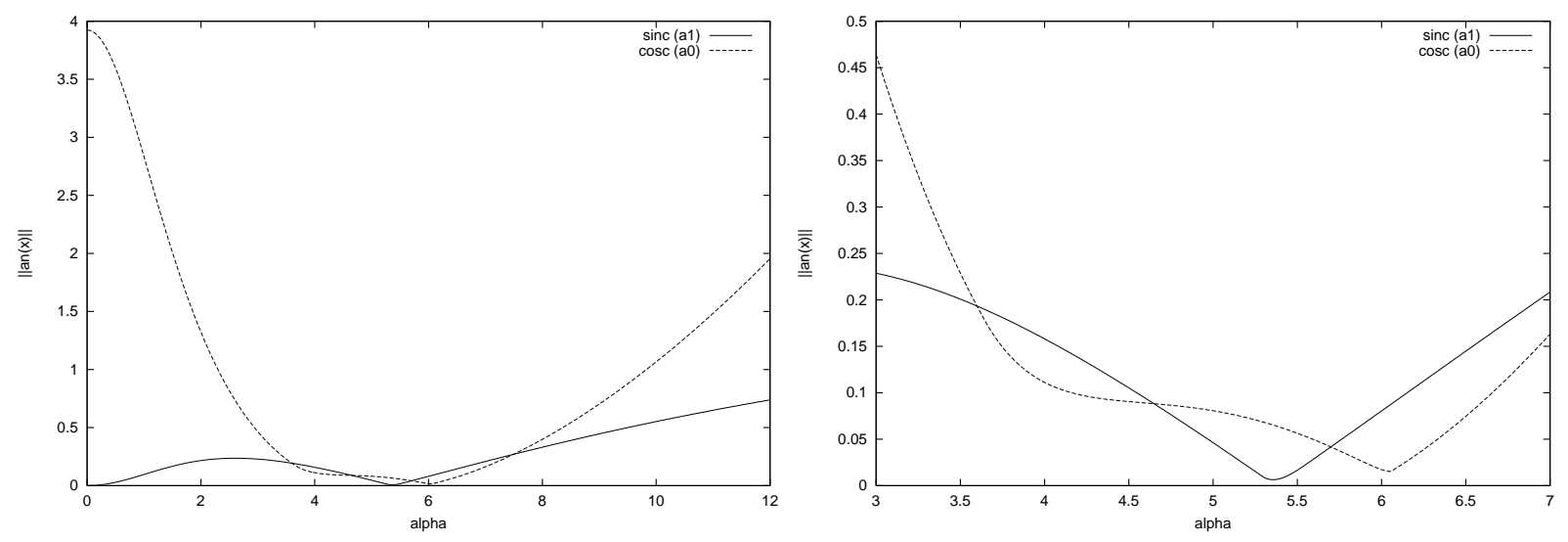

Figure 5: Coefficient plot of Taylor series expansion for Kaiser windowed sinc and cosc with varying parameters and window width two. The right image is a closeup to the minima of these functions.

plotted for the ideal derivative reconstruction filter ( $\operatorname{cosc}$ ) windowed with window width three. In order to appropriately reconstruct the derivative $f^{\prime}(x)$ the coefficient $a_{0}$ should be close to zero in the interval $[0,1]$. On top left, the coefficient plot for the truncated cosc explains the bad result of Fig. 6. Bartlett, Welch, and Parzen window improve the situation but are still far from zero. On top right, Hann, Hamming, and Lanczos window are not much better but the Blackman window shows quite a good result. On bottom left, the coefficient plots for the Kaiser windowed cosc and on bottom right for the Gaussian windowed cosc show, as one could expect, a direct dependence on the choice of the corresponding parameters, $\alpha$ and $\sigma$.

Lets take a closer look at the Kaiser window (a similar argument holds, of course, for the Gaussian window). Choosing $\alpha=2$ obviously is not appropriate for a window width of three as the coefficient plot is quite bad. With $\alpha=4$ the situation gets better, and for $\alpha=8 a_{0}$ is almost zero. With $\alpha=16$ the situation becomes worse again. One can expect a value of $\alpha$ between 8 and 16 being optimal for gradient reconstruction. We will show that such an optimal value exists and how it can be computed.

\section{Optimal parameters for Kaiser and Gaus- sian windows}

As Möller et al. [10] already mentioned, a first derivative reconstruction filter is useless if the first coefficient $a_{0}$ is significantly different from zero. This is the case for the truncated cosc and the results shown in Figs. $6 \mathrm{c}$ and $6 \mathrm{i}$ illustrates this situation. Our goal is to derive optimal parameters, in a purely numerical sense, for Kaiser and Gaussian windowed ideal reconstruction filters.

In order to do this, we compute the $L_{1}$ norm of the coefficient function $a_{n}$, given by

$$
\left\|a_{n}(x)\right\|=\int_{0}^{1}\left|a_{n}(x)\right| \mathrm{d} x
$$

and plot it against the varying parameter of the Kaiser or Gaussian windows. The $L_{1}$ norm was chosen for sake of simplicity, it would, of course, also be possible to use the $L_{2}$ or
$L_{\infty}$ norm. The integral in Eq. 17 can be evaluated numerically. For a specific parameter to be optimal the resulting coefficient error function will have the lowest value. The coefficient error function for the Kaiser windowed cosc (i.e., evaluating Eq. 17 for $a_{0}$ ) with width two for $\alpha$ between zero and twelve can be seen in Fig. 5 on the left. Higher values of $\alpha$ would not be reasonable since the window then already gets too narrow. This coefficient error function indeed has a global minimum, which proves that there is an optimal parameter. The right image in Fig. 5 shows an enlarged part of the neighborhood of this minimum.

We want to apply this concept to find optimal parameters also for the Kaiser and Gaussian windowed sinc filter for function reconstruction. To reconstruct the function itself, the coefficient $a_{0}$ must be one and all others zero. Due to the properties of the Taylor series expansion, the coefficient with most influence will be $a_{1}$. The higher $n$ gets in Eq 15, the lower the contribution of the coefficient. So we plot the $L_{1}$ norm of this coefficient against the varying parameters of Kaiser and Gaussian windowed sinc. The result for the Kaiser windowed sinc of width two can also be seen in Fig. 5 on the left. Surprisingly, we observe that as the parameter $\alpha$ of the Kaiser window approaches zero the coefficient error function approaches zero also. A Kaiser window with $\alpha=0$ is a box function, which would result in a truncation of the sinc function.

However, truncating the sinc filter is commonly known to cause unwanted artifacts, for example ringing [5]. Taking again a look at the error coefficient function of the Kaiser windowed sinc in Fig. 5 we further observe that it takes on another local minimum. The right image in Fig. 5 again shows an enlarged portion of the neighborhood of this minimum. Investigation of $a_{0}$ at this point shows that is is almost constant one, which it is not for $\alpha=0$, so that there is no normalization step necessary. Furthermore, $a_{2}$ at this local minimum is much closer to constant zero than $a_{2}$ for $\alpha=0$ so that we conclude that this local minimum is more appropriate than $\alpha=0$, although $a_{1}$ is not exactly zero. The same holds for the Gaussian window. The only difference is that the Gaussian window approaches constant one (which would result in a rectangular window) as $\sigma$ approaches infinity.

With this method we are now able to compute optimal parameters for Kaiser and Gaussian windowed ideal reconstruction filters, in a strictly numerical sense. We evaluated 


\begin{tabular}{|l|l|c|c|c|c|}
\hline & & \multicolumn{3}{|c|}{ Window width } \\
\cline { 3 - 5 } & & 2 & 3 & 4 & 5 \\
\hline function reconstruction (sinc) & $\alpha$ (Kaiser window) & 5.36 & 8.93 & 12.15 & 15.4 \\
& $\sigma$ (Gaussian window) & 1.11 & 1.33 & 1.46 & 1.63 \\
\hline first derivative reconstruction (cosc) & $\alpha$ (Kaiser window) & 6.05 & 9.28 & 12.5 & 15.5 \\
& $\sigma$ (Gaussian window) & 1.045 & 1.238 & 1.42 & 1.56 \\
\hline
\end{tabular}

Table 1: Optimal values for the parameters for Kaiser and Gaussian windows for function and first derivative reconstruction with window width two, three, four, and five.

values for Kaiser and Gaussian windowed sinc and $\operatorname{cosc}$ of window width two, three, four and five, which are presented in Table 1. Not reflected in this table is the fact that the absolute numerical error is one magnitude smaller for the Kaiser windowed ideal reconstruction filters as compared to the Gaussian or Blackman windowed ideal reconstruction filters.

\section{Results}

To evaluate the results of the last chapters we first tested the reconstruction filters on a standard artificial data set proposed by Marschner and Lobb [6]. We used our framework "SMURF: a SMart sURFace model for advanced visualization techniques" [4] which easily allows to replace reconstruction filters. We used a ray-casting iso-surface extraction algorithm and always used the first derivative of the function reconstruction filter also for first derivative reconstruction. This is according to the scheme proposed by Bentum et al. [1] (with the exception of linear interpolation, where central differences with linear interpolation were used).

Fig. 6a shows the result for central differences and linear interpolation. We clearly observe the smoothing, which is an intrinsic property of this method [2], as the image is too bright. In Fig. 6b, the Catmull-Rom spline and its derivative (i.e., a BC-Spline with $B=0$ and $C=0.5$ [8] or, equivalently, a cardinal spline with $a=-0.5[1])$ were used. We see that the smoothing is reduced but the structure of the artifacts remains. In Fig. 6c truncated sinc and cosc were used. This image shows practically what was discussed theoretically in Chapters 3 and 4. Truncating ideal reconstruction filters is more or less useless.

Figs. 6d - 6f shows images reconstructed with the sinc and cosc filter windowed with the more promising windows from our numerical analysis in Chapter 4. Fig. 6d shows the Blackman window, which is fixed for a certain width (we used a window width of three for this image). The Blackman window gives already quite a good behavior. In Fig. 6e a Kaiser window of width three was used with $\alpha=8.93$ for function reconstruction and $\alpha=9.28$ for first derivative reconstruction, according to Table 1, which further reduces the annoying artifacts present in the previous reconstructions. A Gaussian window of width three with numerically optimal parameters $(\sigma=1.33$ for function reconstruction and $\sigma=1.238$ for first derivative reconstruction) shows some artifacts, especially visible in the highlights, again. This is due to the fact that a window width of three is too narrow for the Gaussian window [14].

The filters were also tested on real world data sets, such as a CT scan of a human head and an MR scan of a human kidney. Figs. $6 \mathrm{~g}-6 \mathrm{l}$ show the results for a close-up of the kidney data set. The same reconstruction filters were used in the same order as for the Marschner-Lobb data set above. Figs. $6 \mathrm{~g}$ and $6 \mathrm{i}$ show again the deficiencies of linear in- terpolation and central differences with linear interpolation and truncated sinc and cosc. Cubic splines (Fig. 6h) show some artifacts especially in the highlights which are removed by the Blackman and Kaiser window (Figs. 6j and 6k). The Gaussian window again introduces artifacts, also, quite notable, in the function reconstruction, due to the window width of three.

Fig. 1 shows the results for the head data set. Linear interpolation for function reconstruction and central differences with linear interpolation for gradient reconstruction were used in the left image. We see that this method introduces quite some smoothing, so that the skull appears quite smooth and visually appealing. However, the close-up below shows that it is not suitable for reconstructing small features. In the middle the Catmull-Rom spline was used both for function reconstruction and gradient reconstruction. We see that the skull exhibits stair case artifacts as they are not smoothed out any more but the close-up clearly exhibits the better reconstruction quality of this filter. On the right we used Kaiser windowed sinc and $\operatorname{cosc}$ with window width three and numerically optimal parameters. Here we can see the better reconstruction especially as the highlights in the close-up appear more sharply. Again, the skull exhibits stair-case artifacts which actually are a visualization of data accuracy. Although the result with central differences is visually more appealing, the Kaiser windowed sinc and cosc are physically more correct.

For further images (also of the other not so good performing windows) please refer to earlier work of the authors [14] and to the web page of this project [15].

\section{Conclusions and Future Work}

We showed that windowing ideal reconstruction filters allows to improve reconstruction quality as compared to the the use of traditional reconstruction methods like linear interpolation or cubic splines. Windowing ideal reconstruction filters also has the advantage that better reconstruction can be achieved by using wider windows, which, however, also increases the computational cost.

From the comparison of some of the more commonly used windows we conclude that the choice of the windowing function is crucial. We showed that some popular windows, like the Lanczos or Hamming window, are not optimal in the sense of numerical accuracy. From our experiments as well as from numerical analysis we found Blackman, Kaiser and Gaussian windows performing best.

Further, we derived numerical optimal values for Kaiser and Gaussian windows, which have a parameter to control their shape. Our analysis of ideal reconstruction filters windowed with these windows using a Taylor series expansion of the convolution sum allowed us to determine optimal values for the parameters of these windows, in a strictly numerical sense. The Kaiser window performs best in this regard 
as the numerical error is smallest. As the Gaussian windows shows some deficiencies for a window width of three, we conclude that the Blackman window is a first good choice which, however, can be improved by using a Kaiser window with numerically optimal parameters.

Continuity conditions, which are very important for the visual appearance of the resulting image [11] have not been addressed and are left for future work. Our experiments showed also that a coupling of function reconstruction and derivative reconstruction improves over-all reconstruction quality. This area deserves further investigation, probably in a way similar to Neumann et al. [12] who use a tight coupling of function and gradient reconstruction based on linear regression.

\section{Acknowledgments}

The work presented in this paper has been funded by the $\mathrm{V}^{\text {is }} \mathrm{M}^{\text {ed }}$ project (http://www.vismed.at/). $\mathrm{V}^{\text {is }} \mathrm{M}^{\text {ed }}$ is supported by Tiani Medgraph, Vienna (http://www.tiani. $\mathrm{com} /$ ) and the Forschungsförderungsfonds für die gewerbliche Wirtschaft, Austria (http://www.telecom.at/fff/). The medical data sets are courtesy of Tiani Medgraph GesmbH, Vienna. Special thanks go to Jiří Hladůvka, Jan Přikryl and Robert F. Tobler for proof reading and valuable hints and discussions.

\section{References}

[1] M. J. Bentum, B. B. A. Lichtenbelt, and T. Malzbender. Frequency Analysis of Gradient Estimators in Volume Rendering. IEEE Transactions on Visualization and Computer Graphics, 2(3):242-254, September 1996.

[2] M. E. Goss. An adjustable gradient filter for volume visualization image enhancement. In Proceedings of Graphics Interface '94, pages 67-74, Banff, Alberta, Canada, May 1994. Canadian Information Processing Society.

[3] J. F. Kaiser and R. W. Schafer. On the Use of the $I_{0}$-Sinh Window for Spectrum Analysis. IEEE Trans. Acoustics, Speech and Signal Processing, ASSP28(1):105, 1980 .

[4] H. Löffelmann, T. Theußl, A. König, and M. E. Gröller. SMURF: a SMart sURFace model for advanced visualization techniques. In Winter School of Computer Graphics '99, pages 156 - 164, Plzen, Czech Republic, 1999. To appear revised as "Smart surface interrogation for advanced visualization techniques" in journal of High Performance Computer Graphics, April/May 2000.

[5] R. Machiraju and R. Yagel. Reconstruction error characterization and control: A sampling theory approach. IEEE Transactions on Visualization and Computer Graphics, 2(4):364-378, December 1996.

[6] S. R. Marschner and R. J. Lobb. An evaluation of reconstruction filters for volume rendering. In R. Daniel Bergeron and Arie E. Kaufman, editors, Proceedings of the Conference on Visualization, pages 100-107, Los Alamitos, CA, USA, October 1994. IEEE Computer Society Press.
[7] E. H. W. Meijering, W. J. Niessen, J. P. W. Pluim, and M. A. Viergever. Quantitative comparison of sincapproximating kernels for medical image interpolation. In C. Taylor and A. Colchester, editors, Medical Image Computing and Computer Assisted Intervention MICCAI'99, pages 210-217, September 1999.

[8] D. P. Mitchell and A. N. Netravali. Reconstruction filters in computer graphics. Computer Graphics, 22(4):221-228, August 1988.

[9] T. Möller, R. Machiraju, K. Müller, and R. Yagel. Classification and local error estimation of interpolation and derivative filters for volume rendering. In Proceedings 1996 Symposium on Volume Visualization, pages 71-78, September 1996.

[10] T. Möller, R. Machiraju, K. Müller, and R. Yagel. Evaluation and Design of Filters Using a Taylor Series Expansion. IEEE Transactions on Visualization and Computer Graphics, 3(2):184-199, 1997.

[11] T. Möller, K. Müller, Y. Kurzion, Raghu Machiraju, and Roni Yagel. Design of accurate and smooth filters for function and derivative reconstruction. In IEEE Symposium on Volume Visualization, pages 143-151. IEEE, ACM SIGGRAPH, 1998.

[12] L. Neumann, B. Csébfalvi, A. König, and M. E. Gröller. Gradient estimation in volume data using 4D linear regression. Technical Report TR-186-2-00-03, Institute of Computer Graphics, Vienna University of Technology, 2000. Accepted for EUROGRAPHICS 2000.

[13] W. H. Press, B. P. Flannery, S. A. Teukolsky, and W. T. Vetterling. Numerical Recipes in C: The Art of Scientific Computing. Cambridge UP, 1988.

[14] T. Theußl. Sampling and reconstruction in volume visualization. Master's thesis, Vienna University of Technology, 2000. http://www.cg.tuwien.ac.at/ ${ }^{\sim}$ theussl/ $\mathrm{DA} /$.

[15] T. Theußl, H. Hauser, and M. E. Gröller. Mastering windows: Improving reconstruction - web page. http://www.cg.tuwien.ac.at/research/vis/ vismed/Windows/.

[16] K. Turkowski. Filters for common resampling tasks. In Andrew S. Glassner, editor, Graphics Gems I, pages 147-165. Academic Press, 1990.

[17] G. Wolberg. Digital Image Warping. IEEE Computer Society Press, 10662 Los Vaqueros Circle, Los Alamitos, CA, 1990. IEEE Computer Society Press Monograph. 


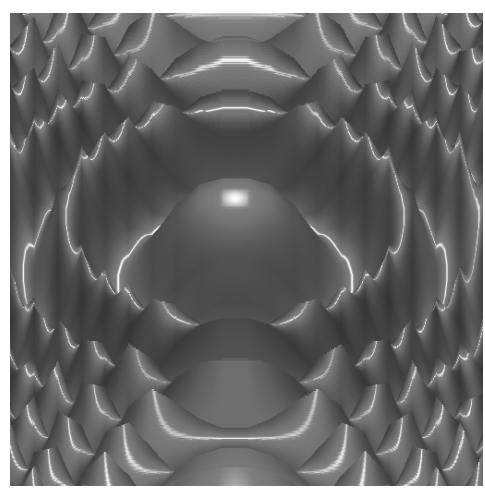

(a)

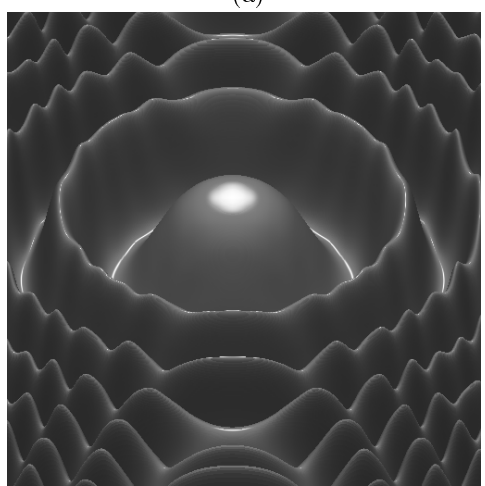

(d)

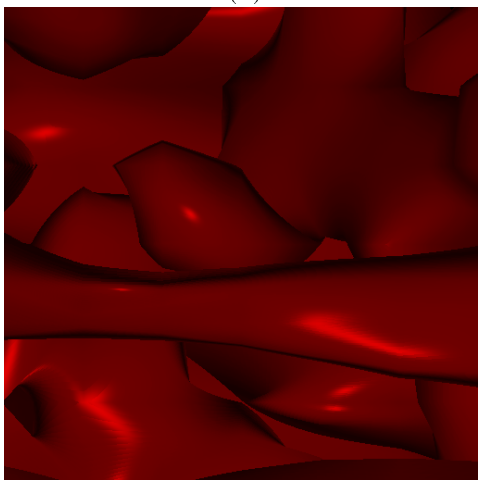

(g)

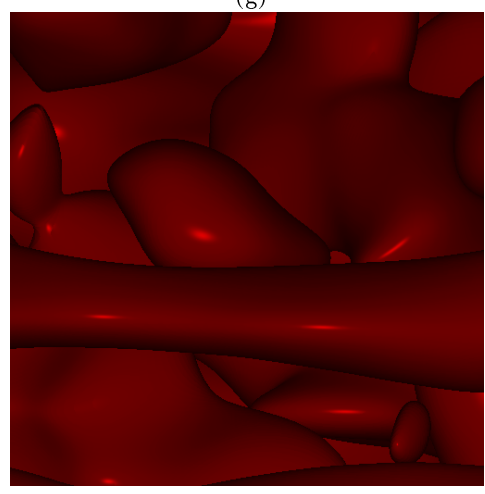

(j)

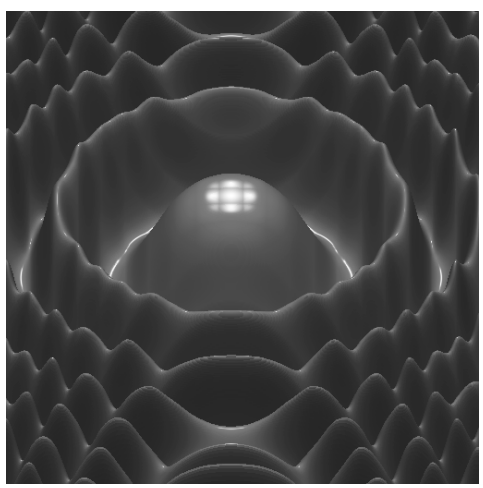

(b)

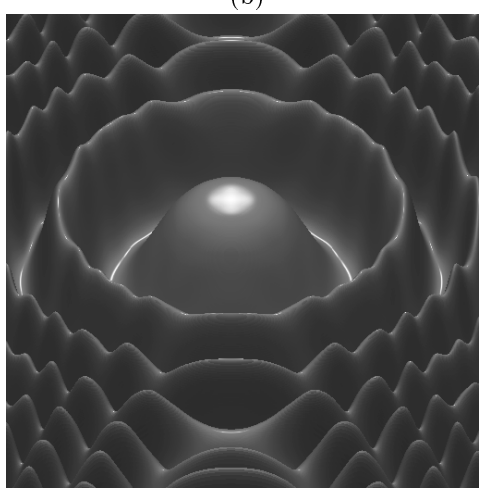

(e)

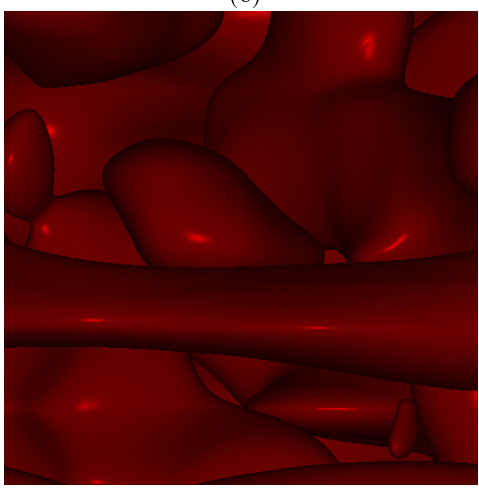

(h)

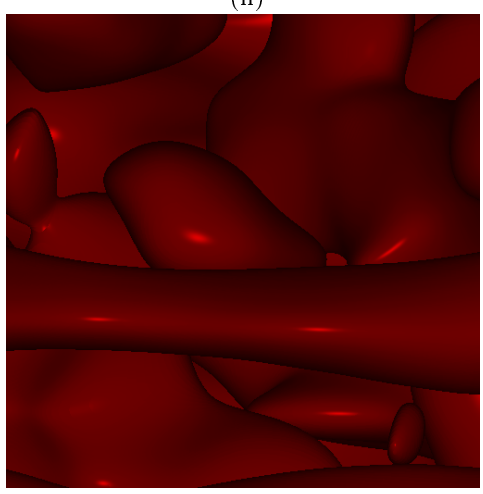

(k)

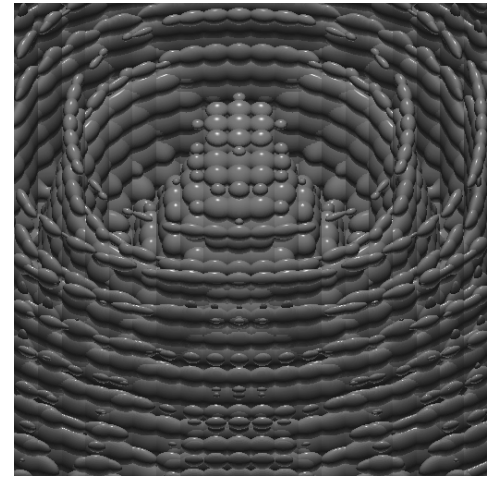

(c)

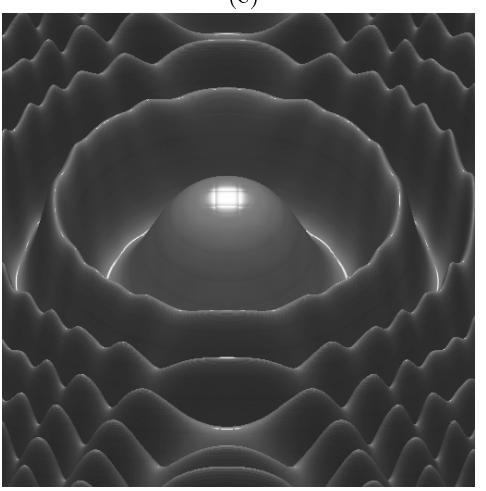

(f)

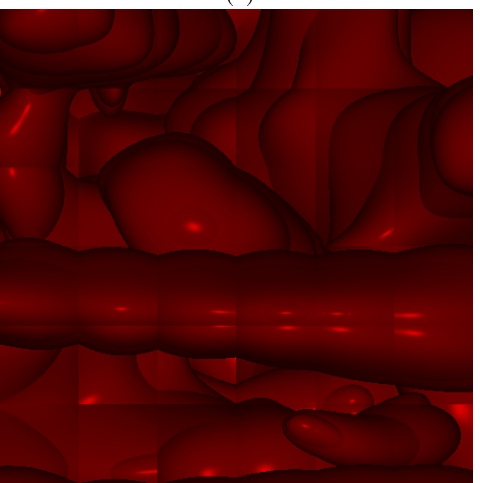

(i)

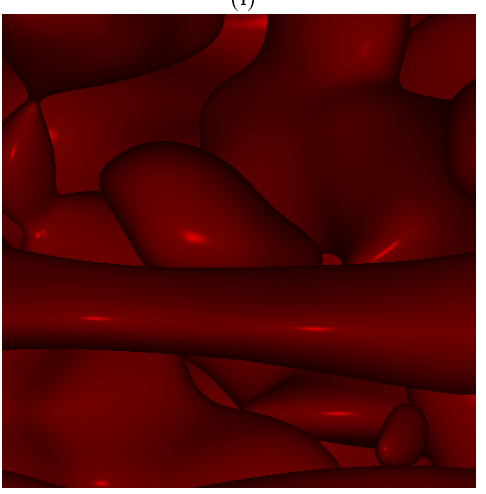

(1)

Figure 6: Marschner Lobb data set $(\mathrm{a}-\mathrm{f})$ and data set of a human kidney $(\mathrm{g}-\mathrm{l})$ reconstructed with $(\mathrm{a}, \mathrm{g})$ linear interpolation and central differences and linear interpolation, $(\mathrm{b}, \mathrm{h})$ Catmull-Rom spline and derivative, $(\mathrm{c}, \mathrm{i})$ truncated (rectangular window) sinc and cosc of width three, $(\mathrm{d}, \mathrm{j})$ Blackman windowed sinc and cosc with window width three, $(\mathrm{e}, \mathrm{k})$ Kaiser windowed sinc and cosc with window width three and numerically optimal parameters and (f,l) Gaussian windowed sinc and cosc with window width three and numerically optimal parameters. 